\title{
Pharmacologic management of pain in patients with Chikungunya: a guideline
}

\author{
Carlos Alexandre Antunes de Brito ${ }^{[1]}$, Ana Karla Arraes von Sohsten ${ }^{[2]}$ \\ Clezio Cordeiro de Sá Leitão[1], Rita de Cássia Coelho Moraes de Brito ${ }^{[3]}$, \\ Lilian David De Azevedo Valadares ${ }^{[4]}$, Caroline Araújo Magnata da Fonte ${ }^{[5]}$, \\ Zelina Barbosa de Mesquita ${ }^{[6]}$, Rivaldo Venâncio Cunha ${ }^{[7],[8]}$, Kleber Luz ${ }^{[9]}$, \\ Helena Maria Carneiro Leão[10], Cecília Moraes de Brito[11] \\ and Lívia Carla Vinhal Frutuoso[12]
}

\begin{abstract}
[1]. Departamento de Medicina Clínica, Universidade Federal de Pernambuco, Recife, Pernambuco, Brasil.
[2]. Instituto de Tratamento da Dor, Real Hospital Português de Beneficência em Pernambuco, Recife, Pernambuco, Brasil. [3]. Departamento de Pediatria, Universidade de Pernambuco, Recife, Pernambuco, Brasil. [4]. Departamento de Reumatologia, Hospital Agamenom Magalhães, Recife, Pernambuco, Brasil. [5]. Departamento de Reumatologia, Hospital Getúlio Vargas, Recife, Pernambuco,

Brasil. [6]. Departamento de Pediatria, Instituto de Medicina Integral Professor Fernando Figueira, Recife, Pernambuco, Brasil.

[7]. Departamento de Infectologia, Universidade Federal de Mato Grosso do Sul, Campo grande, Mato Groso do Sul, Brasil.

[8]. Fundação Oswaldo Cruz, Mato Grosso do Sul, Campo Grande, Mato Grosso do Sul, Brasil. [9]. Departamento de Infectologia, Universidade Federal do Rio Grande do Norte, Natal, Rio Grande do Norte, Brasil. [10]. Escola Superior de Ética e Bioética, Conselho Regional de Medicina de Pernambuco, Recife, Pernambuco, Brasil. [11]. Curso de Graduação em Medicina, Universidade de Pernambuco, Recife, Pernambuco, Brasil. [12]. Programa Nacional de Controle da Dengue, Ministério da Saúde, Brasília, Brasil.
\end{abstract}

\begin{abstract}
From the arrival of Chikungunya virus in the Americas in 2013 until March 2016, approximately two million cases of the disease have been reported. In Brazil, the virus was identified in 2014 and thousands of people have been affected. The disease has high attack rates, infecting $50 \%$ of a population within a few months. Approximately $50 \%$ of infected people develop chronic symptoms lasting for months or years. Joint involvement is the main clinical manifestation of Chikungunya. It is characterized by swelling and intense pain that is poorly responsive to analgesics, both in the acute and chronic phase of the disease. This significantly compromises quality of life and may have immeasurable psychosocial and economic repercussions, constituting therefore, a serious public health problem requiring a targeted approach. Physicians are often not familiar with how to approach the management of pain, frequently prescribing limited analgesics, such as dipyrone, in sub-therapeutic doses. In addition, there are few published studies or guidelines on the approach to the treatment of pain in patients with Chikungunya. Some groups of specialists from different fields have thus developed a protocol for the pharmacologic treatment of Chikungunya-associated acute and chronic joint pain; this will be presented in this review.
\end{abstract}

Keywords: Chikungunya. Pain. Arthralgia. Arbovirus. Treatment.

\section{INTRODUCTION}

From the arrival of the Chikungunya virus (CHIKV) in the Americas in 2013 until March 2016, approximately two million cases of the disease have been reported. In Brazil, the virus was first identified in 2014 and thousands of people have been affected. CHIKV is a single-stranded ribonucleic acid (RNA) virus of the Togaviridae family and the Alphavirus genus with three sub-types (two African and one Asian). The Aedes

\footnotetext{
Correponding author: Dr. Carlos Alexandre Antunes de Brito.

e-mail: cbritoc@gmail.com

Received 15 July 2016

Accepted 23 November 2016
}

aegypti and Aedes albopictus mosquitoes are the main vectors involved in the transmission of CHIKV. Approximately $90 \%$ of individuals infected with CHIKV present with symptomatic infection. The disease may evolve in three phases: acute or febrile (lasting up to 10 days), subacute (11-90 days), and chronic ( $>90$ days). Approximately $50 \%$ of people who experience acute infection develop chronic joint pain that can last months to years. On Reunion Island, it was reported that 3 months after the acute infection, $80-93 \%$ of patients had chronic disease; after 15 months, $57 \%$ did, and after 2 years $47 \%$ of patients had chronic involvement ${ }^{(1)(2)}$.

The joint pain in the different phases of Chikungunya disease causes important physical incapacity that significantly impacts the quality of life of affected patients. In a study by 
Schilte et al. ${ }^{(3)}, 76$ patients with chronic disease were followed for 36 months. Many patients had intermittent arthralgia with recovery and relapse: $45 \%$ had persistent arthralgia, $24 \%$ had initial recovery followed by relapses, and only $31 \%$ showed full recovery by the end of the follow-up period. The study analyzed the impact of the disease on these patients' quality of life. Patients were limited in their ability to lift a chair (62.9\%), walk (54.8\%), pick up objects (54.8\%), open a bottle $(53.2 \%)$, and take a shower (37.1\%). The suffering related to the infection is not limited to pain; a significant portion of patients experience mental health and sleeping disorders and mood swings ${ }^{(3)(4)}$. The economic impact of a CHIKV outbreak on the Reunion Islands between 2005 and 2006, considering the cost of medical consultations, therapeutic treatment and the cost of work time lost due to illness or pain, was estimated at $€ 34$ million per year ( $\mathrm{R} \$ 119$ thousand reais), corresponding to $€ 250.00$ per patient per year ${ }^{(3)}$.

The chronic joint complaints in the chronic phase can assume the patterns of other chronic inflammatory diseases. Javelle et al. assessed 159 cases of chronic Chikungunya with more than two years of evolution. Of these cases, $112(70 \%)$ had features of a chronic rheumatic inflammatory disease, with 40 of these cases meeting the clinical and radiologic criteria for rheumatoid arthritis, 33 for spondyloarthritis, and 21 for undifferentiated polyarthritis. None had a history of rheumatologic disease.

The pain, besides being intense, is poorly responsive to analgesics. In a study by Andrade et al. ${ }^{(5)}$ involving 106 patients with Chikungunya, the intensity of pain was assessed by the visual analog scale (VAS): The average was $5.8 \pm$ 2.1. The authors reported that many patients did not respond to the analgesics prescribed, with only $26 \%$ experiencing pain relief (relief of $>70 \%$ of the pain). Furthermore, the pain was neuropathic in nature in $18.9 \%$ of patients. There are few studies or guidelines in the literature regarding the approach to pain treatment. The majority of published studies and guidelines are limited to stating the drugs used in pain treatment, which include: dipyrone, paracetamol, non-steroidal anti-inflammatories (NSAIDs), corticosteroids, codeine, and morphine. The use of methotrexate, chloroquine, and sulfasalazine has also been reported in patients with chronic pain $^{(1)(6)(7)(8)(9)(10)}$. A significant proportion of physicians are not familiar with how to approach the treatment of pain in Chikungunya, with frequent prescriptions limited to analgesics such as dipyrone, often in sub-therapeutics doses. There is also a lack of systematized practice of using staggered therapy according to the intensity of the pain and reassessment after failure/success of the drug administered. Given this need, a group of specialists from different fields collaborated to produce a protocol for the pharmacologic treatment of acute and chronic Chikungunya-associated joint pain; it is presented in this review.

\section{THE PATHOPHYSIOLOGY OF THE PAIN OF CHIKUNGUNYA}

Despite the improved understanding of joint damage associated with infection by alphaviruses, the cause of persistent symptoms remains unresolved. The inflammatory response of the host, the presence of viral products in macrophages and joint tissues, and auto-immune process may be involved in the pathogenesis. Different joint manifestations during different phases of Chikungunya fever have been described in the literature, including arthralgia, inflammatory arthritis, synovitis, enthesitis, tenosynovitis, and bursitis ${ }^{(11)}$. Experimental models of arthritis induced by alphaviruses suggest that the pathogenesis is the result of a combination of direct tissue and cell damage caused by viral replication and indirect immune activation responses in the target tissues ${ }^{(12)}$. Different cytokines, chemokines, and other inflammatory mediators are produced; these are related to the intensity of inflammation in the acute phase; are involved in the recruitment of macrophages, natural killer cells, and $\mathrm{T}$ lymphocytes to the site of viral replication; and cause dysregulation of inflammation in this stage, leading to the expression of other inflammatory proteins responsible for damaging the joint in the chronic phase ${ }^{(12)(13)(14)(15)}$.

Viral persistence could also be a cause of chronic joint disease in a subgroup of patients. The presence of immunoglobulin M (IgM) antibodies detected months after the acute infection suggests persistence of the virus or its antigens; this may perpetuate the inflammatory process in the joints ${ }^{(16)}$. In an outbreak in Italy, Moro et al. reported that IgM was detected in $52 \%(131 / 249)$ of patients within 5 months of the contracting disease and in $13.2 \%(30 / 227)$ with 12 months of disease evolution ${ }^{(17)}$. Other risk factors associated with progression to a chronic form include age $>35$ years, marked joint impairment in the acute phase, and pre-existing prior joint disease $\mathrm{e}^{(4)(9)(18)}$. Some patients have polymorphisms of the human leukocyte antigens (HLA) that are associated with rheumatic diseases, such as HLA-27. These HLA polymorphisms may be involved in the pathogenesis of the disease ${ }^{(11)(12)}$.

A sub-group of patients present with neuropathic-type pain $^{(5)}$. Neuropathic pain syndromes are caused by lesions in or dysfunction of the nervous system; they do not directly depend on an inflammatory processes, but involve specific changes in central and peripheral nociceptive processes ${ }^{(19)}$. Therefore, it seems that the mechanisms are multifactorial and may different between patients. A clear understanding of the pathophysiologic mechanisms in patients with Chikungunya will have a direct bearing on the choice of ideal therapeutic agent to relieve the pain.

\section{CLINICAL AND LABORATORY DIAGNOSIS OF CHIKUNGUNYA-ASSOCIATED ARTHRALGIA/ARTHRITIS}

A suspected case of Chikungunya is defined as a fever of sudden onset, higher than $38.5^{\circ} \mathrm{C}$, and intense arthralgia or arthritis not explained by other conditions in a person resident of or having visited an endemic or epidemic area up to two weeks before the beginning of the symptoms, or with an epidemiologic link to a confirmed case. The differential diagnosis includes infections caused by other virus or bacteria, as well as rheumatologic diseases that have fever as a symptom. Other arbovirus that are frequent and may circle at the same time and in the same epidemic areas, mainly dengue and Zika, must be borne in mind. In areas where it circulates, Mayaro virus, 
an alphavirus that has arthralgia as symptom of infection, must also be considered ${ }^{(1)}$.

The laboratory diagnosis of Chikungunya in the first 5-7 days includes techniques for the detection of CHIKV genome [reverse transcription polymerase chain reaction (RT-PCR) and/or real-time RT-PCR] and viral isolation in cell cultures. Specific anti-CHIKV IgM antibodies are formed during the first week of the disease and may be detected from the seventh day on ${ }^{(1)}$. Specific examination and testing are only routinely performed in the periods between epidemics in order to detect the first cases and identify the start of an epidemic. However, once sustained transmission has been established, not all patients require laboratory confirmation. In this context, laboratory investigation is reserved for patients with alarm signs, severe cases, those with atypical manifestations, or in cases of difficult differential diagnosis. The diagnosis may be confirmed based on clinical-epidemiological criteria ${ }^{(1)}$.

In the sub-acute and chronic phases of arthralgia, a history of having had an episode of acute fever with associated edema and joint pain during an epidemic period (clinical-epidemiologic criteria) dispenses with the need to perform serologic tests in patients that seek assistance for the treatment of pain. For patients not responding to initial analgesic therapy, serologic tests for Chikungunya [IgM, immunoglobulin $\mathrm{G}(\operatorname{IgG})]$ and tests in search of other etiologies must be performed, at the discretion of the specialist. Non-specific tests, such as a complete blood count, may be requested in the acute phase and biochemical tests such as transaminases, creatinine, and electrolytes may be necessary in at-risk patients or those who are severely ill( ${ }^{(1)}$.

In the chronic phase, for patients that do not respond to treatment, autoantibodies (rheumatoid factor; anti-citrullinated protein antibody; and anti-nuclear antibodies) and molecular markers (HLA-B27) must be requested, according to the disease suspected, as the differential diagnosis includes chronic inflammatory arthritides ${ }^{(20)}$.

\section{STAGGERED APPROACH TO MANAGEMENT OF ACUTE AND CHRONIC PAIN}

Treatment involves all phases of the disease and cannot focus only on the sub-acute and chronic stages; it should be effective even during the first few days of experiencing symptoms. Neuroscientific studies have established that inadequately treated acute pain is one of the main causes of chronic pain, which then triggers other symptoms such as depression, fatigue, and sleeping disorders. This natural history is no different when it comes to Chikungunya. Therefore, an effective approach to control the pain is needed. This will also serve to decrease the duration of this clinical disease. The flowcharts contained in this document address the management of pain in different stages of the disease, aiming to increase the effectiveness of the available pharmacologic therapies (Figure 1, Figure 2, Figure 3, Figure 4, Figure 5, Figure 6, Figure 7 and Figure 8).

\section{Pain measurement}

To institute a pain management protocol, it is essential to have a tool that allows the measurement of pain, and that can

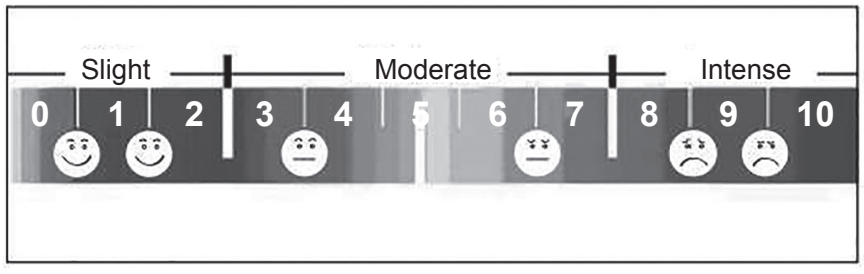

FIGURE 1. The visual analog scale combined with a face scale.

transform subjective data into objective data; this enables proper assessment and management. Without this, it becomes difficult to determine if a treatment is necessary, if the prescription is effective, or if it should be interrupted or substituted ${ }^{(21)}$.

There are several validated tools to use; however, the VAS is one of the simplest and can be applied by any health professional (Figure 1 and Table 1). Another scale used widely in Brazil is the face scale. It can be used with small children and elderly people with cognitive deficits. However, despite the simplicity of the tool, it is important to remember that pain is a subjective perception and therefore, has a relationship with social, cognitive, and psychological aspects. It is necessary that while measuring pain, the patient should be informed of the importance of being truthful, and that there is an approach for each type of pain and numbering in order to avoid methodologic failures; patients often report a very high level of pain (9-10 in VAS) in fear of not receiving analgesics if the numbers are too low.

\section{PHARMACOLOGIC TREATMENT}

\section{Analgesics}

Dipyrone and paracetamol: these are good analgesics when administered in appropriate doses and at regular intervals (Figures 2, 3, 7 and Figure 8). Dipyrone can be prescribed at $30-50 \mathrm{mg} / \mathrm{kg} /$ dose, 6 hourly. Paracetamol can be prescribed at $500-750 \mathrm{mg}, 6$ hourly, but the total daily dose should not exceed at $4 \mathrm{~g}$ because of possible hepatotoxicity. If the patient's pain is perceived as mild (VAS 1-3) one of these two drugs should be prescribed, always in fixed, regular doses and never when necessary. In cases of moderate pain (VAS 4-6) both drugs should be prescribed together, at fixed times, and in an alternating fashion (i.e., the patient will take an analgesic dose every three hours).

Opioids: the opioid drugs are potent and safe analgesics, especially in cases of acute pain. Adverse effect monitoring is required, and the patient should be warned about adverse effects. In the doses usually prescribed by non-specialists in pain management, the risk of respiratory depression is very low; drowsiness and lethargy are the preceding warning signs (Figures 2, 3, 7, and Figure 8). Tramadol is a good choice when suspicious of a neuropathic component of intense pain as, besides its action on opioid receptors, it acts as an antagonist of NMDA ( $N$-methyl-D-aspartate) receptor that are involved in chronic pain. Tramadol should be used in a dose of 50-100mg, 6 hourly. Codeine is an opioid that should be prescribed in a dose of 30mg every 6 hours and can be used with other analgesics. 
TABLE 1

FLACC scale, for children aged two months to seven years.

\begin{tabular}{llll}
\hline & & & \multicolumn{2}{c}{ Points } \\
\cline { 2 - 4 } Category & \multicolumn{1}{c}{$\begin{array}{l}\text { No special expression or smile } \\
\text { Face }\end{array}$} & $\begin{array}{l}\text { Grimace or occasional frowning eyebrows, } \\
\text { introversion, disinterest }\end{array}$ & Frequent chin trembling, tight jaw \\
Legs & Normal or relaxed & Agitated, restless, tense & Kicking or stretched legs \\
Activity & $\begin{array}{l}\text { Quiet, in a normal position, moving } \\
\text { Cry }\end{array}$ & Stirs and contorts, moving back and forth, tense & Curved, stiff or sudden movements \\
Console & No crying (awake or asleep) & Moans or whines; occasional complaining & $\begin{array}{l}\text { Continuous cry, screaming or with the hiccups; } \\
\text { complaining frequently }\end{array}$ \\
\hline
\end{tabular}

Observe children for 5 minutes, points 0-2 are assigned up to the maximum total of 10 points (intense pain). FLACC: Face, Legs, Activity, Cry, Consolability scale.

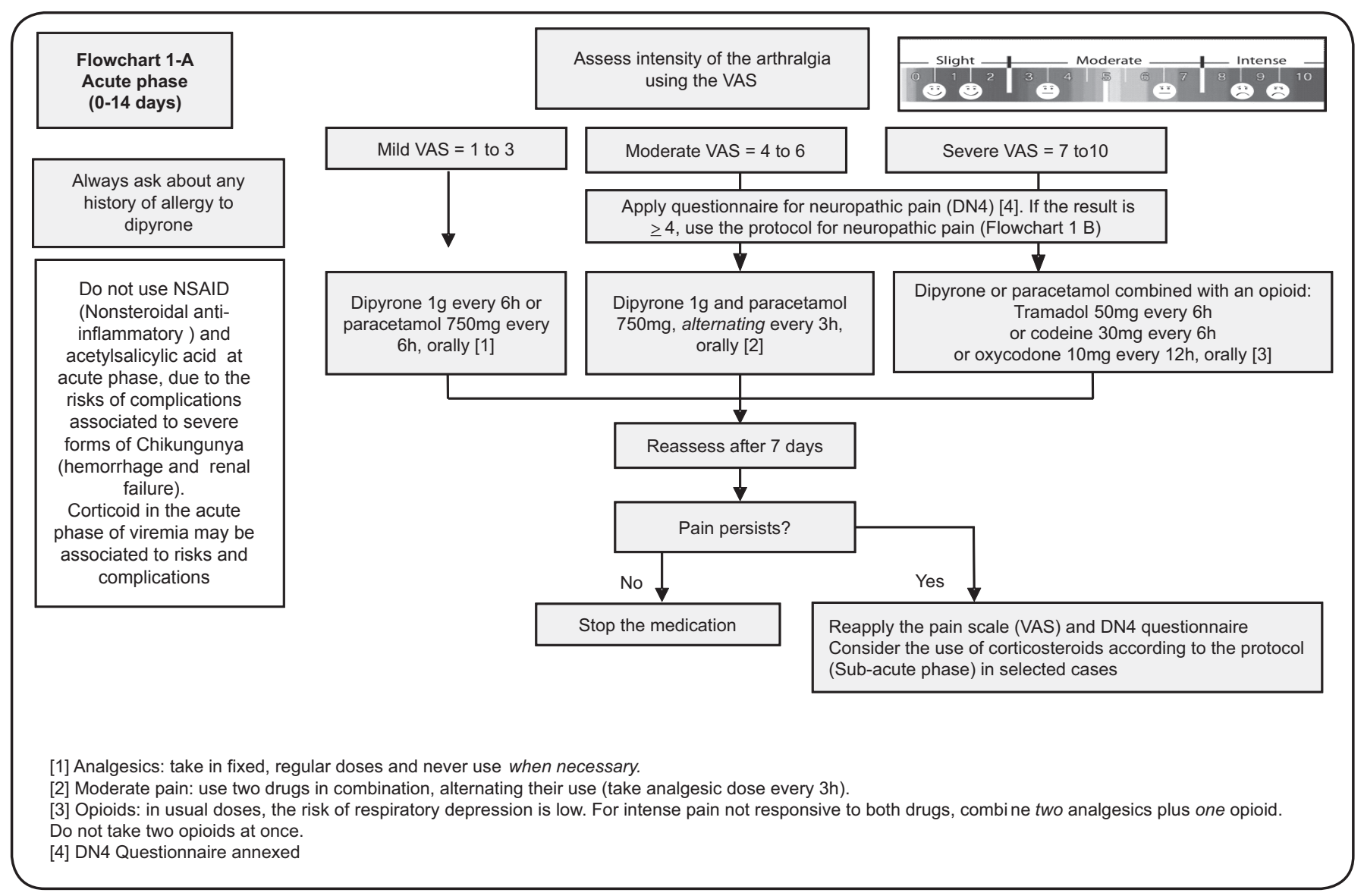

FIGURE 2. Flowchart 1-A. Treatment of arthralgia in acute phase of Chikungunya in adults. VAS: visual analog scale; DN4: Douleur Neuropathique 4; NSAIDs: non-steroidal anti-inflammatories. 


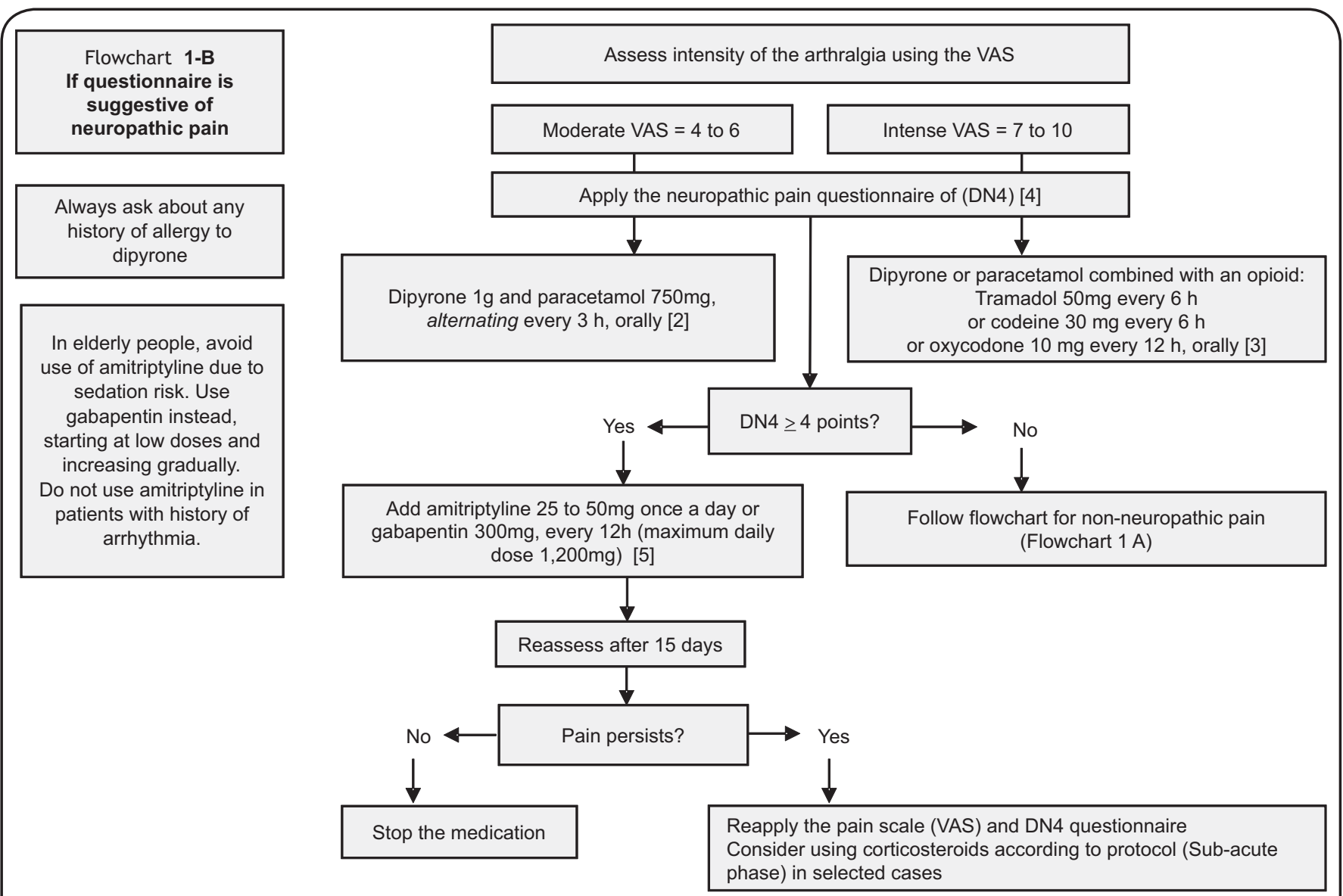

[4] DN4 questionnaire annexed.

[5] The anti-depressants and anti-convulsants may need up to 2 weeks to show an effect.

FIGURE 3. Flowchart 1-B. Treatment of neuropathic pain in adults with Chikungunya. VAS: visual analog scale; DN4: Douleur Neuropathique 4.

Oxycodone is a synthetic opioid that has an extended-release formulation, allowing its prescription at intervals of 12 hours. The recommended dose is 10-20mg, 12 hourly (Figure 2).

NSAIDs and acetylsalicylic acid: despite the inflammatory component in the physiopathogenesis of the disease, NSAIDs should not be used due to the risk of complications associated with severe forms of Chikungunya (hemorrhage and renal insufficiency). Similarly, acetylsalicylic acid should not be used due to the risk of bleeding. In the acute phase, the differential diagnosis includes infection with other arbovirosis, such as Dengue, that are associated with thrombocytopenia; in such situations the use of these drugs is also contraindicated ${ }^{(1)}$.

Corticosteroids: in the acute phase of viremia, corticosteroids can be associated with increased risks and complications.

\section{Anti-convulsant and anti-depressant drugs}

Neuropathic pain may be present if joint pain does not respond to the usual analgesics, making it necessary to add these therapeutic classes to treatment, after clinical confirmation (Figure 4 and Figure 7). In patients who experience severe and prolonged pain, it is recommended that the signs and symptoms of neuropathic pain be screened for using the Douleur Neuropathique 4 (DN4) questionnaire (Table 2). This is composed of two oral questions and two questions requiring a physical examination, totaling ten answers. If four or more answers are positive, the patient probably has a neuropathic component to their pain ${ }^{(22)}$.

For these patients, the use of drugs that modulate the excitatory activity of the nervous system, such as amitriptyline and gabapentin, are indicated. Studies suggest that these drugs act by modulation of the lesion or dysfunction of the nervous system, reducing the nervous activity responsible for the maintenance of neuropathic pain. In elderly people, amitriptyline may lead to sedation, making it preferable to take gabapentin, starting in low doses. Patients with a medical history of arrhythmias should not take amitriptyline (opt for gabapentin). The use of gabapentin should be carefully considered and the dose should be optimized if the individual presents with sedation and drowsiness.

\section{The role of corticosteroids (drugs of sub-acute phase)}

The use of corticosteroids is indicated for the disease in its sub-acute phase in patients with moderate to severe pain 
TABLE 2

DN4- Questionnaire used to diagnose neuropathic pain.

Please complete this questionnaire by ticking one answer for each item in the 4 questions below:

\section{Patient interview}

Question 1: Does the pain have one or more of the following characteristics?

1. Burning

2. Painful cold

3. Electric shocks

Question 2: Is the pain associated with one or more of the following symptoms in the same area?

Yes

No
4. Tingling
5. Pins and needles
6. Numbness
7. Itching

\section{Examination of the patient}

Question 3: Is the pain located in an area where the physical exam may reveal one or more of the following characteristics?

Yes

8. Hypoesthesia to touch

9. Hypoesthesia to prick

Question 4: In the painful area, can the pain be caused or increased by:

10. Brushing

\section{Score}

Zero (0): For each negative item. One (1): For each positive item.

The total score is calculated as the sum of the 10 items. The cut-off value for the diagnosis of neuropathic pain is a total score $\geq 4 / 10$. 


\begin{tabular}{|c|}
\hline Flowchart 1-C \\
Acute phase (0-14 days), \\
Attend to urgently. \\
\hline Do not use NSAIDs \\
(nonsteroidal anti- \\
inflammatories) or \\
acetylsalicylic acid in the \\
acute phase due to the risk \\
of complications \\
associated with severe \\
forms of Chikungunya \\
(hemorrhage and renal \\
failure). \\
Corticosteroid use in the \\
acute phase of viremia can \\
be associated with risks \\
and complications \\
urgency service \\
emergency care units or \\
intense pain, polyarticular, \\
or disabling pain, may \\
need intravenous (IV) \\
medication in the \\
prescribed orally, and \\
phe at the ambulatory \\
service according to \\
The medications can be \\
\hline
\end{tabular}

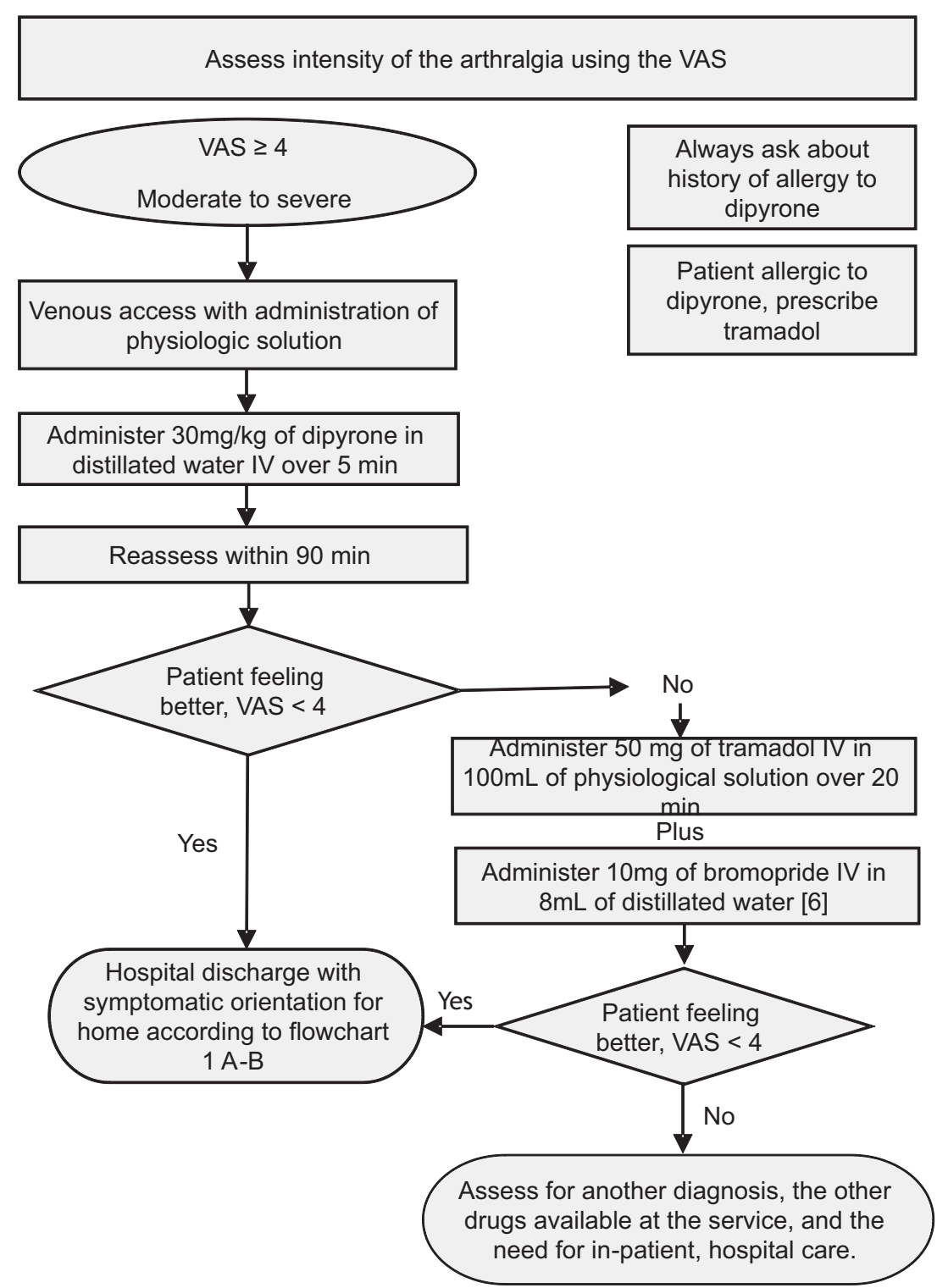

[6] Nausea may be present, due to tramadol or the infection; thus, administer $10 \mathrm{mg}$ of bromopride with the tramadol.

FIGURE 4. Flowchart 1-C. Treatment of arthralgia in the acute phase of Chikungunya in adults in urgency/ emergency units. VAS: visual analog scale; DN4: Douleur Neuropathique 4; NSAIDs: non-steroidal anti-inflammatories.

based on the VAS scale (Figure 5 and Figure 7). The standard medication for oral use is prednisone, that has anti-inflammatory effects when taken in low doses; its anti-inflammatory effects predominate at a dose $\leq 0.5 \mathrm{mg} / \mathrm{kg}$ per day. Given at intermediate doses ( $>0.5$ to $<1 \mathrm{mg} / \mathrm{kg}$ per day), its effect lies between antiinflammatory and immunosuppressive actions, whereas in higher doses ( $\geq 1 \mathrm{mg} / \mathrm{kg}$ per day) its immunosuppressive action predominates, independent of its anti-inflammatory action. For the treatment of pain, the dose is $0.5 \mathrm{mg} / \mathrm{kg}$ per day as a single dose taken in the morning. An adequate response to the treatment is best assessed by improvement in the ability to walk without assistance and satisfactory pain control. If the treatment response is adequate, continue at the recommended dose until the full resolution of the joint pain.

In the event of complete remission of pain, continue taking the recommended dose for 3-5 more days. If there is no relapse, start weaning by decreasing the dose by $5 \mathrm{mg} /$ day every 7 days.

The initial dose may be maintained for up to 21 days, the average time during which there is usually no risk of inducing adrenal insufficiency.

During the weaning phase, if pain recurs, return to the previous dose and try to repeat weaning only five days after 
Flowchart 2 Sub-acute phase (after 10-14 days)

Always ask about a history of allergy to anti-inflammatories

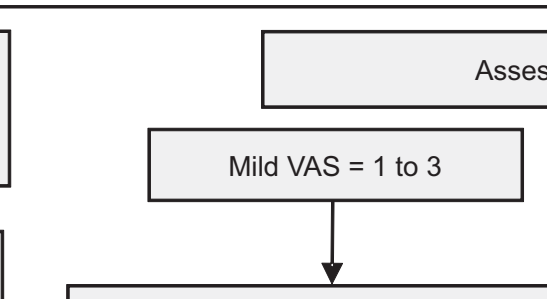

Non-steroidal anti-inflammatories [7] Ibuprofen $600 \mathrm{mg}$ every $8 \mathrm{~h}$, orally

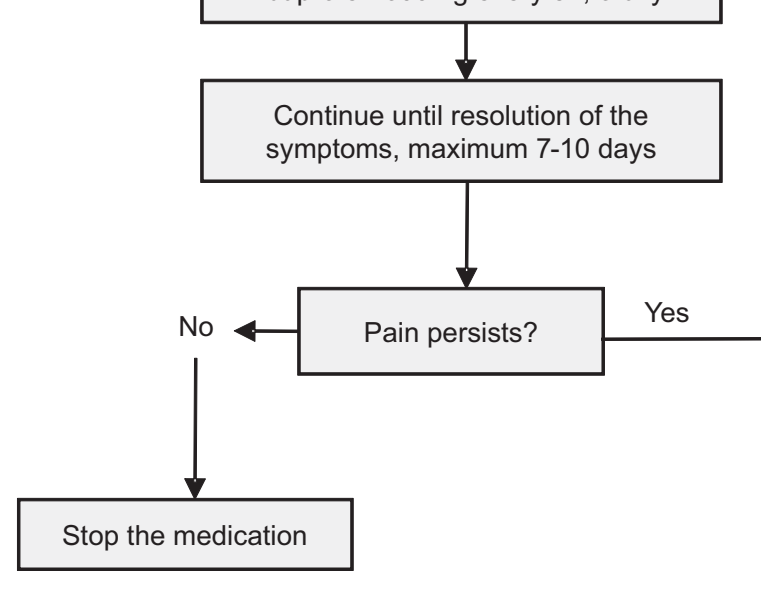

Assess intensity of the arthralgia using the VAS

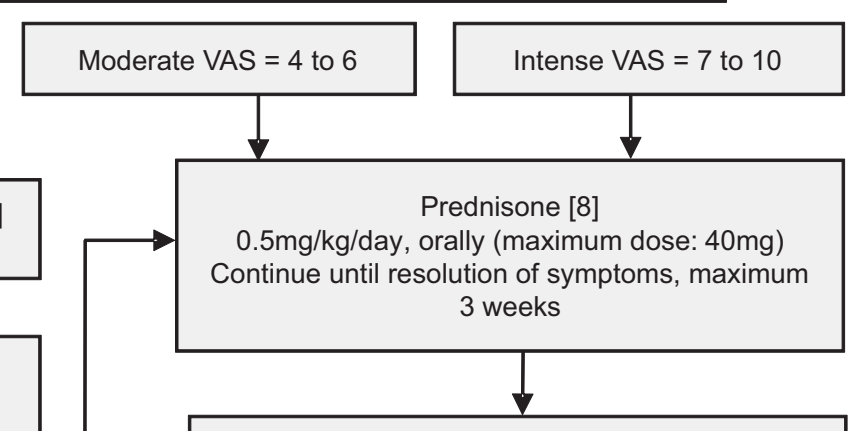

After resolution of the symptoms, maintain dose for $3-5$ days and begin weaning by $5 \mathrm{mg} /$ day every 7 days

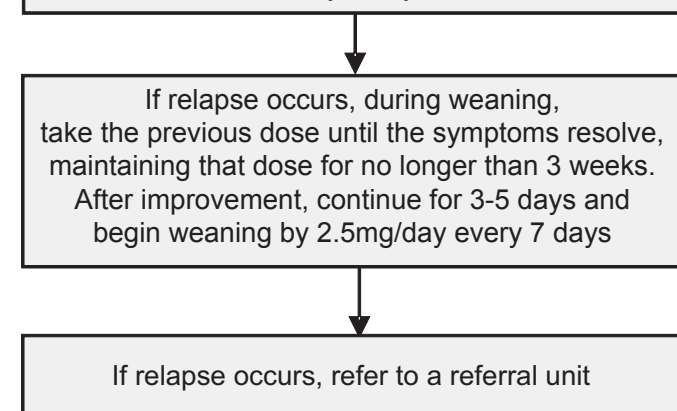

[7] NSAIDs: only to be used after the acute phase (>10-14 days). Be attentive to side effects. Renal function must be assessed in elderly people and in those with comorbidities, prior to starting treatment. Be alert for the higher risk for chronic degenerative diseases in patients such as the elderly, those with diabetes, peptic ulcer disease, nephropathy, liver disease, and cardiopathy, among others.

[8] Until the onset of corticosteroid action, prescribe analgesics according to protocol (Flowchart 1A-B)

- Anti-inflammatory dose of corticosteroid is $\leq 0.5 \mathrm{mg} / \mathrm{kg}$ per day

- Exclusion criteria for the use of corticosteroids: Patients with diabetes, difficult to control hypertension, history of fracture due to documented osteoporosis, bipolar mood disorder, chronic renal failure on dialysis, Cushing's syndrome, obesity grade III, arrhythmias, and coronary artery disease.

- There is no risk of inducing adrenal insufficiency if used for $\leq 21$ days.

- Corticosteroids may be prescribed for patients in the chronic phase if not yet used.

FIGURE 5. Flowchart 2. Treatment of arthralgia in the sub-acute phase of Chikungunya in adults. VAS: visual analog scale; NSAIDs: non-steroidal antiinflammatories.

symptom resolution. The weaning must be slower, with a decrease of $2.5 \mathrm{mg}$ /day every seven days.

Exclusion criteria for the use of corticosteroids: the use of corticosteroids is contraindicated in patients with diabetes, difficult to control hypertension, previous documented osteoporotic fracture, bipolar mood disorder, chronic renal failure on dialysis, Cushing's syndrome, obesity grade III, arrhythmias, and coronary artery disease.

\section{Hydroxychloroquine, sulphasalazine, methotrexate (chronic phase drugs)}

The medical care of patients with chronic Chikungunya must take place in referral units with professionals qualified to assist these patients. The medications used in this phase of treatment - hydroxychloroquine, sulphasalazine, and methotrexate - have adverse effects related to their therapeutic classes and require clinical and laboratory monitoring before and during use. They must be prescribed by qualified professionals (Figure 6 and Figure 8). There are a limited number of publications regarding the use of these drugs in the chronic phase of Chikungunya. Most studies involved a small number of patients, and different methodologies were used; thus, it is currently impossible to draw conclusions regarding the efficacy of these drugs, or to assess the superiority of different therapies in patients with chronic Chikungunya ${ }^{(23)(24)(25)(26)(27)(28)}$. The use of these drugs is based on extrapolation from their use in the treatment of chronic rheumatic diseases ${ }^{(29)(20)(30)}$.

The chronic phase of Chikungunya is defined as continuity of symptoms for more than three months after the onset of the disease; it may last for many years. Usually, the disease progresses to the cure without sequelae, either spontaneously or after treatment. However, the patient may present with persist 


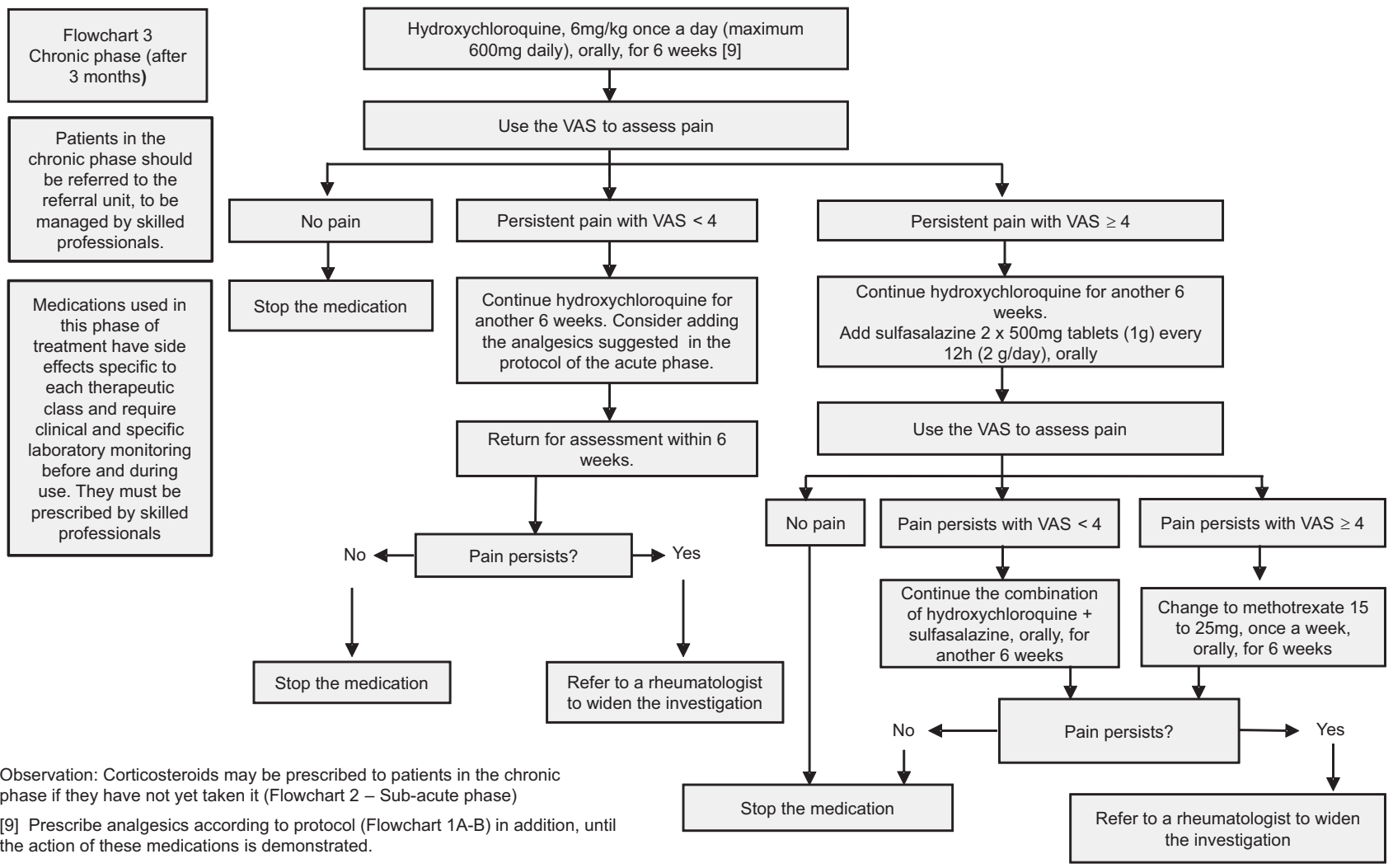

FIGURE 6. Flowchart 3 . Treatment of arthralgia in the chronic phase of Chikungunya in adults. VAS: visual analog scale.

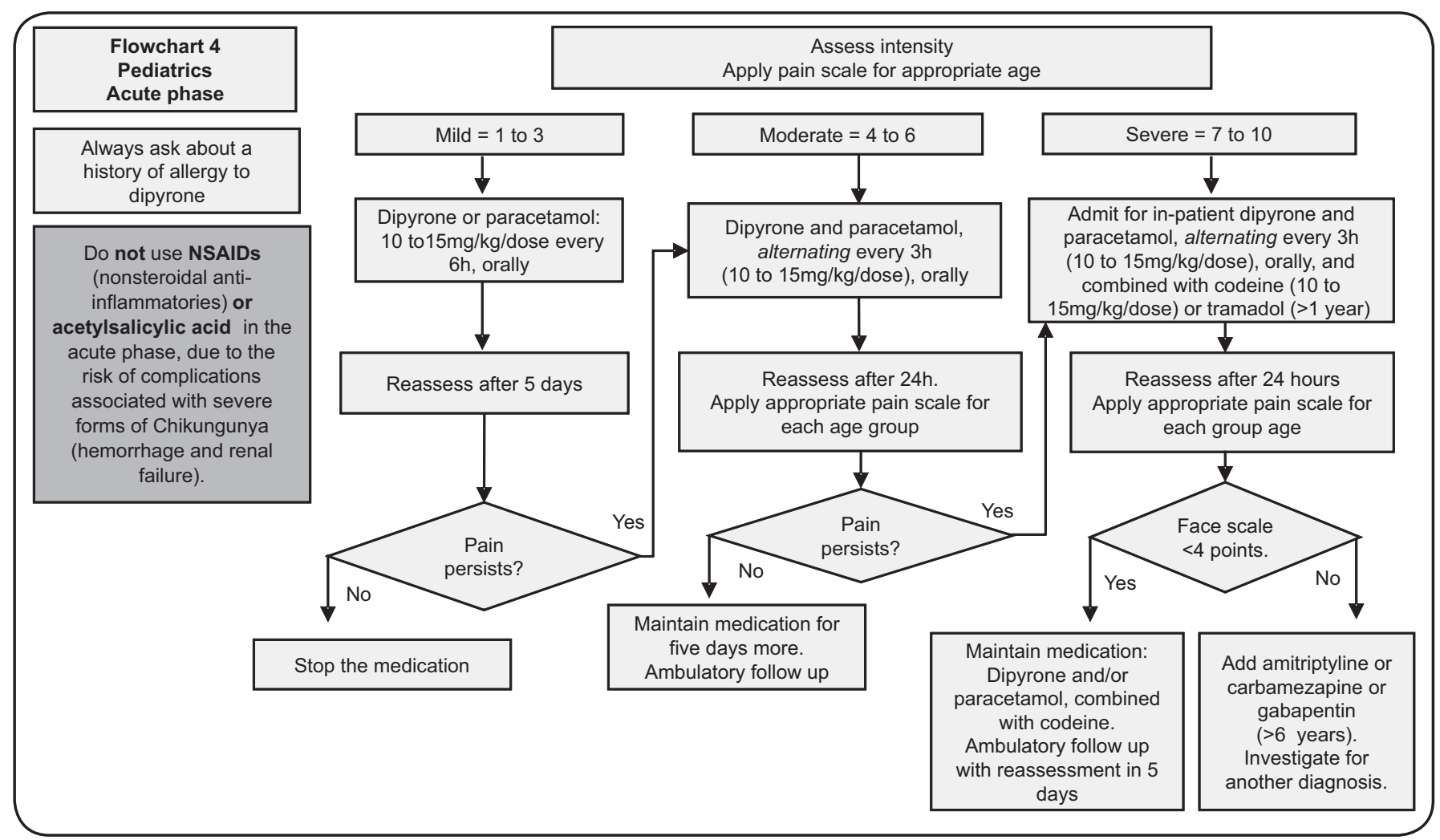

FIGURE 7. Flowchart 4. Treatment of arthralgia in the acute phase of Chikungunya in children. NSAIDs: non-steroidal anti-inflammatories. 


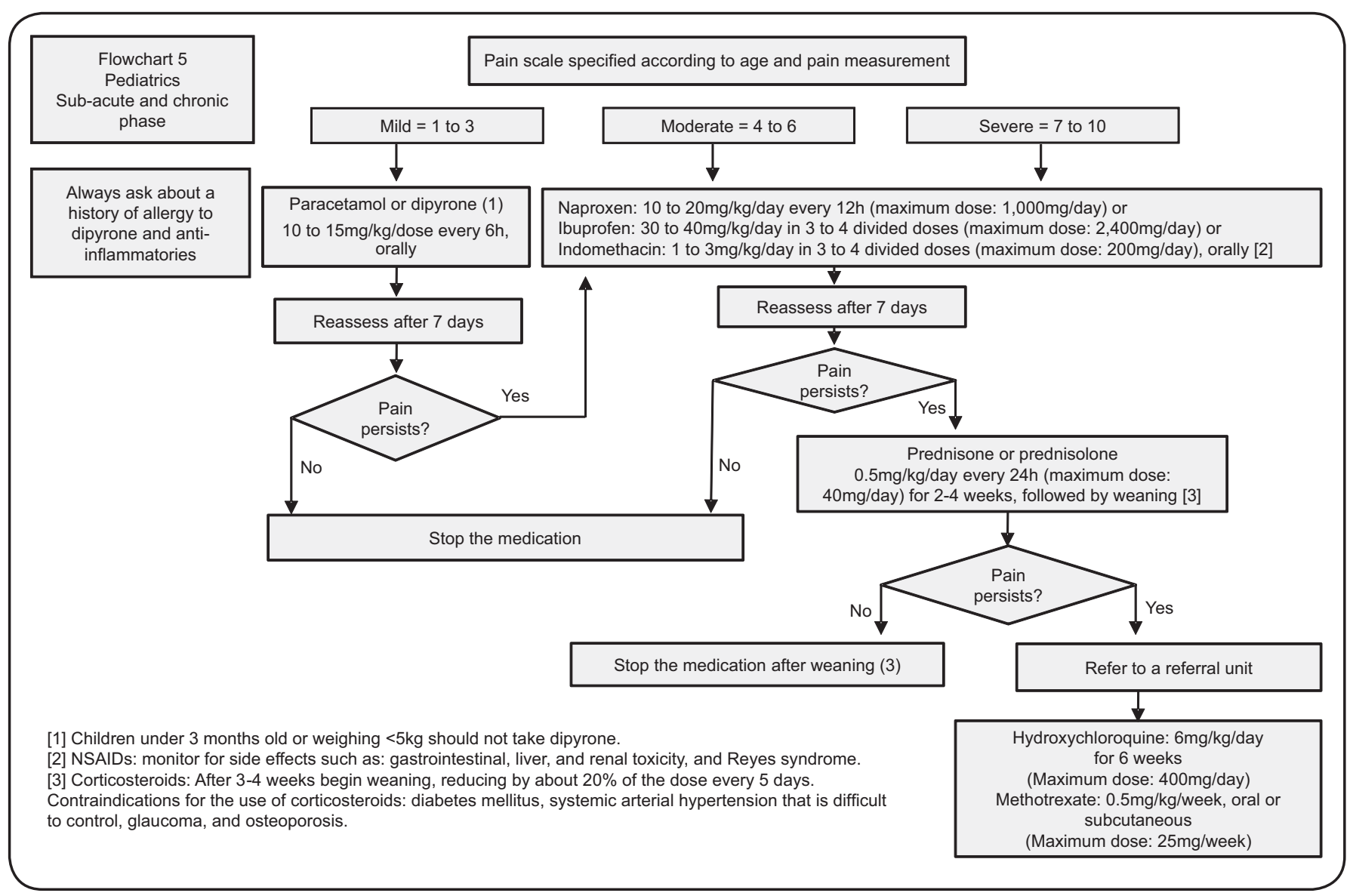

FIGURE 8. Flowchart 5. Treatment of arthralgia in the sub-acute and chronic phases of Chikungunya in children. NSAIDs: non-steroidal anti-inflammatories.

global or joint symptoms due to the worsening of a degenerative or inflammatory condition. This presentation may manifest as pronounced tenosynovitis or as a distal edematous polyarthralgia ${ }^{(20)}$. Despite the lack of data comparing the efficacy of methotrexate and hydroxychloroquine in patients with Chikungunya, we have chosen to recommend hydroxychloroquine as the treatment of first choice in the chronic phase. This recommendation is based on hydroxychloroquine's anti-inflammatory effects in the control of arthritis and musculoskeletal pain ${ }^{(30)}$ and its potential antiviral action $^{(31)}$, but most of all, it is a safer drug than methotrexate in the context of being prescribed by non-specialists.

In the chronic stage, therapy can be initiated with hydroxychloroquine in a dose of $6 \mathrm{mg} / \mathrm{kg} / \mathrm{day}$ (maximum dose: $600 \mathrm{mg} /$ day) taken orally for a period of six weeks. After six weeks, the patient should be reassessed using the VAS. If the patient is pain-free, the treatment should be discontinued. If the VAS is less than four, the treatment should be maintained for a further six weeks, and analgesics may be added, as suggested in the protocol for the acute phase (Figure 2). If the pain persists with a VAS greater than or equal to four, sulphasalazine should be added at a dose of $1,000 \mathrm{mg}$ (two $500 \mathrm{mg}$ tablets) in 12 hourly intervals, with the use of hydroxychloroquine extended for six weeks more. This combination is known to be effective in controlling the joint symptoms in patients refractory to the treatment used previously ${ }^{(29)}$.
The patient should return for assessment after six weeks, when the VAS scale is applied again. If after this period the patient is without pain, the medication should be stopped. If the VAS is less than four, hydroxychloroquine and sulphasalazine should be continued for another six weeks. If the VAS is greater than or equal to four, the combination of hydroxychloroquine and sulphasalazine should be replaced by methotrexate $(2.5 \mathrm{mg}$ tablet), at an initial dose of $10 \mathrm{mg} /$ week, orally; this dose may be may be increased to $25 \mathrm{mg} /$ week. Folic acid should always be given in combination with methotrexate, at a dose of $5 \mathrm{mg} /$ week, taken the day after methotrexate is taken ${ }^{(20)(32)}$.

If after six weeks there is persistence of pain, the patient should be referred to a rheumatologist to widen the diagnostic investigation and introduce other therapies. Be attentive to the main side effects of methotrexate, including hepatotoxicity, myelotoxicity, and gastrointestinal effects. The dose must be adjusted in patients with impaired renal function, and it is contraindicated in patients with a creatinine clearance $<30 \mathrm{~mL} /$ $\min$.

The main side effects of hydroxychloroquine include visual disturbances, hematologic abnormalities, and alopecia. The risk of adverse events is higher in patients with glucose-6-phosphate dehydrogenase deficiency, liver failure, porphyria, psoriasis, myopathies, or cardiomyopathy. 


\section{Non-pharmacologic treatment}

Physiotherapy should be considered, beginning in the acute phase of Chikungunya. It may be combined with cryotherapy as an analgesic measure. It should also be prescribed in the subacute and chronic phases, aiming to minimize the osteoaticular damage, consequently enabling rehabilitation. Physiotherapy should be requested equally in the three phases of disease; however, it should be a priority in the sub-acute and chronic phases. The manufacture and use of orthoses as an adjuvant measure may be required; this may achieve more rapid relief of pain and, above all, may help to prevent muscular atrophy consequent to the disuse of the compromised joint. Other therapies, such as acupuncture, may be used in the sub-acute and chronic phases. It is worth mentioning that psychological support is important in all phases of the disease, especially to relieve the sadness and suffering arising from the state of pain and chronic swelling during a long period of consequent illness.

\section{CONCLUSION}

Chikungunya epidemics, with the high attack rate of CHIKV, affect a large number of people in a short period of time, producing not only acute cases, but also a high number of chronic cases. Pain, the most frequent clinical manifestation of Chikungunya, is difficult to control, compromising the quality of life of affected patients. It may have immeasurable psychosocial and economic repercussions, constituting a serious public health problem that requires a targeted approach. There are few protocols for managing the pain caused by Chikungunya; hence, it was necessary to produce a guideline to diagnose and appropriately manage patients with Chikungunya. It is important to emphasize that in addition to medical treatment, the approach to the management of patients with Chikungunya requires the involvement of multidisciplinary teams. General physicians, specialists, nurses, pain specialists, physiotherapists, social workers, and healthcare managers are required to institute these guidelines and to organize services to manage patients with different levels of complexity.

\section{Acknowledgments}

The authors thank the Ministério da Saúde, Brazil and the Conselho Regional de Medicina de Peranambuco, Brazil for their valuable cooperation with this Project.

\section{Conflict of interest}

The author declares that there is no conflict of interest.

\section{REFERENCES}

1. Ministério da Saúde. Secretaria de Vigilância em Saúde. Febre de Chikungunya manejo clínico. Brasília: Ministério da Saúde; 2015. p. $1-28$.

2. Borgherini G, Poubeau P, Jossaume A, Gouix A, Cotte L, Michault A, et al. Persistent arthralgia associated with chikungunya virus: a study of 88 adult patients on reunion island. Clin Infect Dis 2008; 47:469-475.
3. Schilte C, Staikovsky F, Couderc T, Madec Y, Carpentier F, Kassab $\mathrm{S}$, et al. Chikungunya virus-associated long-term arthralgia: a 36-month prospective longitudinal study. Plos Negleted Trop Dis 2013; 7 e2137. doi: 10.1371/journal.pntd.0002137.

4. Ramachadran V, Kaur P, Kanagasabai S, Vadivoo S, Murhekar M. Persistent arthralgia among Chikungunya patients and associated risk factors in Chennai, South India. J Postgrad Med 2014; 60:3-6.

5. de Andrade DC, Jean S, Clavelou P, Dallel R, Bouhassira D. Chronic pain associated with the Chikungunya fever: long lasting burden of an acute illness. BMC Infect Dis 2010; 10:31.

6. Staikowsky F, Le Roux K, Schuffenecker I, Laurente P, Grivard P, Develay A, et al. Retrospective survey of Chikungunya disease in Réunion Island hospital staff. Epidemiol Infect 2008; 136:196-206.

7. World Helth Organization. (WHO). Guidelines for prevention and control of Chikungunya fever. South-East Asia: WHO; 2009. p. 1-43.

8. Organización Panamericana de la Salud (OPS). Preparación y respuesta ante la eventual introducción del virus chikungunya en las Américas. Washington: OPS; 2011. p. 1-148.

9. Ali Ou Alla S, Combe B. Arthritis after infection with Chikungunya virus. Best Pract Res Clin Rheumatol 2011; 25:337-346.

10. Caglioti C, Lalle E, Castilletti C, Carletti F, Capobianchi MR, Bordi L. Chikungunya virus infection: an overview. New Microbiol 2013; 36:211-227.

11. Waymouth HE, Zoutman DE, Towheed TE. Chikungunya-related arthritis: case report and review of the literature. Semin Arthritis Rheum 2013; 43:273-278.

12. Assunção-Miranda I, Cruz-Oliveira C, Da Poian AT. Molecular mechanisms involved in the pathogenesis of alphavirus-induced arthritis. BioMed Res Int 2013; 2013:1-11. ID 973516.

13. Hoarau JJ, Jaffar Bandjee MC, Krejbich Trotot P, Das T, Li-Pat-Yuen G, Dassa B, et al. Persistent chronic inflammation and infection by Chikungunya arthritogenic alphavirus in spite of a robust host immune response. J Immunol 2010; 184:5914-5927.

14. Ng LF, Chow A, Sun YJ, Kwek DJC, Lim PL, Dimatatac F, et al. IL-1 $\beta$, IL-6, and RANTES as Biomarkers of Chikungunya severity. PLoS One 2009; 4:e4261. doi 10.1371/journal.pone.0004261

15. Chow A, Her Z, Ong EKS, Chen JM, Dimatatac F, Kwek DJC, et al. Persistent arthralgia induced by Chikungunya virus infection is associated with interleukin- 6 and granulocyte macrophage colonystimulating factor. J Infect Dis 2011; 203:149-157.

16. Malvy D, Ezzedine K, Mamani-Matsuda M, Autran B, Tolou $\mathrm{H}$, Receveur MC, et al. Destructive arthritis in a patient with Chikungunya virus infection with persistent specific IgM antibodies. BMC Infect Dis 2009; 9:200. doi: 10.1186/1471-2334-9200.

17. Moro ML, Grilli E, Corvetta A, Silvi G, Angelini R, Mascella F, et al. Long-term chikungunya infection clinical manifestations after an outbreak in Italy: a prognostic cohort study. J Infect 2012; 65:165172.

18. Yaseen HM, Simon F, Deparis X, Marimoutou C. Identification of initial severity determinants to predict arthritis after chikungunya infection in a cohort of French gendarmes. BMC Musculoskelet Disord 2014; 15:249. doi: 10.1186/1471-2474-15-249.

19. Bouhassira D, Attal N, Alchaar H, Boureau F, Brochet B, Bruxelle $\mathrm{J}$, et al. Comparison of pain syndromes associated with nervous or somatic lesions and development of a new neuropathic pain diagnostic questionnaire (DN4). Pain 2005; 114:29-36.

20. Simon F, Javelle E, Cabie A, Bouquillard E, Troisgros O, Gentile G, et al. French guidelines for the management of chikungunya (acute 
and persistent presentations). November 2014. Med Mal Infect 2015; 45:243-263.

21. Souza FAEF. Dor: o quinto sinal vital. Rev Latino-am Enferm 2002; 10:446-447.

22. Haanpää M, Attal N, Backonja M, Baron R, Bennett M, Bouhassira $D$, et al. NeuPSIG guidelines on neuropathic pain assessment. Pain 2011; 152:14-27.

23. De Lamballerie X, Boisson V, Reynier JC, Enault S, Charrel RN, Flahault A, et al. On chikungunya acute infection and chloroquine treatment. Vector Borne Zoonotic Dis 2008; 8:837-839.

24. Pandya S. Methotrexate and hydroxychloroquine combination therapy in chronic chikungunya arthritis: a 16 week study. Indian J Rheumatol 2008; 3:93-97.

25. Padmakumar B, Jayan JB, Menon RMR, Krishnankutty B, Payippallil R, Nisha RS. Comparative evaluation of four therapeutic regimes in chikungunya arthritis: a prospective randomized parallel-group study. Indian J Rheumatol 2009; 4:94-101.

26. Chopra A, Saluja M, Venugopalan A. effectiveness of chloroquine and inflammatory cytokine response in patients with early persistent musculoskeletal pain and arthritis following chikungunya virus infection. Arthritis Rheumatol 2014; 66:319-326.
27. Ganu MA, Ganu AS. Post-chikungunya chronic arthritis - our experience with DMARDs over two year follow up. J Assoc Physicians India 2011; 59:83-86.

28. Rosario V, Munoz-Louis R, Valdez T, Adames S, Medrano J, Paulino I, et al. Chikungunya infection in the general population and in patients with rheumatoid arthritis on biological therapy. Clin Rheumatol 2015; 34:1285-1287.

29. Arroyo-Ávila M, Vilá LM. Rheumatic manifestations in patients with Chikungunya infection. P R Health Sci J 2015; 34:71-77.

30. Ben-Zvi I, Kivity S, Langevitz P, Shoenfeld Y. Hydroxychloroquine: from malaria to autoimmunity. Clin Rev Allerg Immunol 2012; 42:145-153.

31. Thiberville SD, Moyen N, Dupuis-Maguiraga L, Nougairede A, Gould EA, Roques P, et al. Chikungunya fever: epidemiology, clinical syndrome, pathogenesis and therapy. Antiviral Res 2013; 99:345-370.

32. Javelle E, Ribera A, Degasne I, Gaüzère BA, Marimoutou C, Simon F. Specific management of post-chikungunya rheumatic disorders: a retrospective study of 159 cases in Reunion Island from 2006-2012. Plos Negleted Trop Dis 2015; 9:e0003603. doi: 10.1371/journal. pntd.0003603. 